often occurs. On the basis of these results, the authors conclude that nearly $10 \%$ of SIDS cases can be attributed to genetic arrhythmogenic disorders. As LQTS is characterized by QT prolongation, electrocardiographic screening during the third or fourth week of life might identify infants at risk of SIDS.

Taken together, these studies provide strong support for the hypothesis that a proportion of autopsy-negative sudden deaths are caused by LQTS, and suggest that electrocardiogrambased screening programs might facilitate the identification of individuals at risk of sudden death, affording the opportunity for preventative intervention.

Original articles Tester DJ and Ackerman MJ (2007) Postmortem long QT syndrome genetic testing for sudden unexplained death in the young. J Am Coll Cardiol 49: 240-246 Arnestad M et al. (2007) Prevalence of long-QT syndrome gene variants in sudden infant death syndrome. Circulation 115: $361-367$

\section{Disproportionate transmission of long QT syndrome to females}

Congenital long QT syndrome (LQTS), a rare cardiac disorder, is associated with syncope, and with sudden cardiac death from ventricular arrhythmias. Inheritance is predominantly autosomal dominant, but an increased susceptibility has been observed in women. Guicheney and co-workers have ascertained that there is an imbalance between the sexes in transmission of the mutated alleles that cause the syndrome.

The investigators retrospectively studied 484 nuclear families with type 1 LQTS and 269 families with type 2 LQTS, all of European origin. Of the 234 clinically diagnosed probands, 159 (68\%) were female. The number of offspring with type 1 or type 2 mutations (in the potassium-channel genes KCNQ1 and $K C N H 2$, respectively) was higher than expected at 870/1534 (57\%), and displayed a predominance of females - 476/870 (55\%) of the carriers of an LQTS allele were female $(P=0.005)$. While male carriers of the type 1 LQTS allele demonstrated a classic Mendelian pattern of transmission to their offspring $(P=0.57)$, female transmission was markedly increased $(P<0.001)$. Transmission was distorted among both mothers and fathers carrying an allele for the type 2 syndrome $(P=0.13$ and $P=0.009$, respectively).
There are several physiological factors that could predispose women to LQTS, such as longer QT intervals and a possible increased risk of certain cardiac events. Importantly, this study also shows favored transmission of mutant alleles to daughters, and the authors believe this could be a substantial contributing factor to the observed female predominance of the syndrome.

Original article Imboden $\mathrm{M}$ et al. (2006) Female predominance and transmission distortion in the long-QT syndrome. N Engl J Med 355: 2744-2751

\section{0-year stroke trends in the Framingham Study}

The past half century has seen considerable advances in the treatment of stroke; reports of temporal trends in stroke incidence, risk, severity, and mortality have, however, been mixed. Now, Carandang et al. have evaluated data from 9,152 individuals who participated in the original (recruited in 1948) and offspring (enrolled in 1971) cohorts of the Framingham Study. Three consecutive periods were compared: 1950-1977, 1978-1989, and 1990-2004.

A total of 1,030 first clinical strokes were observed over 174,917 person-years of followup. Age-adjusted incidences of first clinical stroke declined with time in both men and women (from 7.6 to 5.3 per 1,000 person-years in men, $P=0.02$ for trend; and from 6.2 to 5.1 per 1,000 person-years in women, $P=0.01$ for trend). At age 65 years, 10-year risk of stroke was significantly lower in 1990-2004 than in 1950-1977 for both men and women $(P=0.01$ and $P=0.04$, respectively); lifetime risk tended to be lower in men $(P=0.11)$, but not in women $(P=0.61)$. Over the study period, 30-day mortality also declined in men, but not in women, possibly reflecting the increasing age of women at the time of first stroke. No trends were detected for severity of stroke or post-stroke disability.

The authors conclude that advances in care over the past 50 years have reduced the prevalence of most risk factors for stroke and lowered its incidence, but improvements in lifetime risk, severity, and short-term mortality rates are still required.

Original article Carandang R et al. (2006) Trends in incidence, lifetime risk, severity, and 30-day mortality of stroke over the past 50 years. JAMA 296: 2939-2946 